\title{
Ecología de la anidación de Crocodylus acutus (Reptilia: Crocodylidae) en Bahía Portete, La Guajira, Colombia
}

\author{
John Jairo Gómez-González ${ }^{1}$ 2*, Juan Carlos Narváez-Barandica ${ }^{3}$, Lina Báez ${ }^{4}$ \& \\ Edgar Patiño-Flórez ${ }^{1}$ \\ 1. Fundación Hidrobiológica George Dahl, Carrera 43 No. 47 - 32 Local 2, Barranquilla, Colombia; \\ potosflavus@gmail.com, avinicio1989@gmail.com \\ 2. Fundación Ornitológica del Atlántico - ORNIAT, Transversal 1D Sur No. 71D - 46, Soledad, Atlántico, Colombia. \\ 3. Laboratorio de Genética Molecular, Universidad del Magdalena, Carrera 32 No 22 - 08, Santa Marta, Colombia; \\ jenarvaezb@yahoo.es \\ 4. Carbones del Cerrejón Limited, Albania, La Guajira, Colombia; linabaez@yahoo.com \\ * Correspondencia
}

\section{Recibido 31-VIII-2015. Corregido 14-VIII-2016. Aceptado 14-IX-2016.}

\begin{abstract}
Nesting ecology of Crocodylus acutus (Reptilia: Crocodylidae) in Bahia Portete, La Guajira, Colombia. C. acutus is cataloged in critical danger in Colombia. We studied its population at Bahia Portete, in order to survey the nesting activities and to obtain valuable information for a conservation management program. This study was undertaken with the participation of the Wayuü community using the Agreement Conservation Model, and took place during July 2007, August 2008, June and August 2009, and March to August 2010 and 2011. Sampling surveys were made by the use of transects along the coast, in order to find nests. For each nesting area found, we recorded the nests biometrics, eggs and hatchlings, fertility of the eggs and reproductive events. We explored a total of $55.12 \mathrm{~km}$, and determined four nesting areas. Colonial nesting and the environmental variables were factors that affected the hatchling success. From the total of nests found, $37 \%$ were oriented towards North, and $48 \%$ were built next to Stenocereus griseus. Juyui island substrate was mostly constituted by sand $(71.9 \%, 71.44 \%)$. The average number of eggs by nest was $28.42 \pm 6.63$, the largest egg diameter was $71.84 \pm 3.54 \mathrm{~mm}$, the average nest weight was $81.54 \pm 9.99 \mathrm{~g}$, and the hatchlings presented an average length of $25.47 \pm 1.16 \mathrm{~cm}$. For this specific site in Colombia, the reproductive period begins in March and ends in August. The few areas of nesting and the $95 \%$ fertility suggest the performance of a conservation management program for this species at Bahía Portete. Rev. Biol. Trop. 65 (1): 211-228. Epub 2017 March 01.
\end{abstract}

Key words: crocodiles, Crocodylus acutus, Bahía Portete, nesting, reproduction.

Crocodylus acutus es una de las 23 especies de cocodrilos descritas a escala mundial (Ross, 1998). Sus poblaciones se encuentran distribuidas desde el sur de la Florida, en el litoral Atlántico y Pacífico de los países de Centro América, las Antillas mayores y los países de Sur América, Colombia, Venezuela, Ecuador y Perú (Rodríguez-Melo, 2000a; Thorbjarnarson, 2010). Ocupa una alta diversidad de hábitats correspondientes a ríos, zonas costeras, pantanos y marismas conformados por agua dulce, salobre, salina e hipersalinas (Rueda-Almonacid et al., 2007; Thorbjarnarson, 2010; De La
Ossa-Lacayo, De La Ossa V, Fajardo Patiño, \& Morales-Betancourt, 2013).

A escala internacional esta especie se encuentra catalogada como "Vulnerable" por la Unión Internacional para la Conservación de la Naturaleza (Ponce-Campos, Thorbjarnarson, \& Velasco, 2012) y en Colombia en la categoría de "Peligro Crítico" en el libro rojo nacional (Rodríguez-Melo, 2002). El último censo sobre la situación de la especie en Colombia, desarrollado entre 1994 y 1997, encontró una población fragmentada y con la presencia de seis subgrupos poblacionales prioritarias 
para el desarrollo de programas de conservación, por estar conformadas con representantes de todas las edades. Estos subgrupos están localizados en: río Man (Antioquia), ciénaga la Caimanera (Sucre), caño Grande (Córdoba), ciénagas de Puerto Badel (Bolívar), cuenca del río Dibulla (Guajira) y Bahía Portete (La Guajira) (Rodríguez-Melo, 2000b). Luego, a estos grupos se les sumó la población del río Catatumbo (Ulloa-Delgado, 2011). Para Bahía Portete, la población se consideró como una Unidad de Conservación de Crocodílidos (Thorbjarnarson et al., 2006), debido a los altos valores de abundancia y al estado de conservación de sus hábitats.

La población de cocodrilos de esta bahía ha sido objeto de estudios esporádicos. Abadia (1995; 1996) evaluó previamente la reproducción y amenazas de la población. Determinó una densidad relativa entre 0.09 y 0.5 ind./ $\mathrm{km}$ en $22 \mathrm{~km}$ de distancia; sólo observó dos ejemplares de más de $182 \mathrm{~cm}$ de longitud total; y estimó una población de 140 individuos. Con relación a la reproducción, concluyó que la anidación inicia entre abril y mayo, con un tamaño de la nidada entre 19 y 41 huevos, y construidas en las playas rocosas de la bahía. Además, identificó como principal amenaza, su consumo por parte de los indígenas Wayuü y la comercialización de las pieles hacia Venezuela. Debido a estos antecedentes, la empresa Carbones del Cerrejón Limited desarrolló entre 2007 y 2011 el Programa de Recuperación Poblacional de C. acutus en Bahía Portete con el objetivo de determinar aspectos demográficos y describir su proceso de anidación (De la Hoz, Patiño, Gómez, Mejía, \& Báez, 2007). Por lo tanto, en este manuscrito se analizaron los datos generados en el monitoreo para: i) determinar las áreas de anidación; ii) identificar los factores que influyen en la anidación; iii) determinar las características de la ecología de la anidación; y iv) de la biología reproductiva, para definir el período de anidación en este sector de Colombia y ayudar con un plan de conservación para esta población.

\section{MATERIALES Y MÉTODOS}

Área de estudio: Bahía Portete es la más grande de una serie de bahías ubicadas en el sector más septentrional de la península de La Guajira en Colombia (Fig. 1). Está comunicada con el mar Caribe por una boca de aproximadamente dos kilómetros hacia la parte noroccidental (Solano, 1994). Se localiza a los $12^{\circ} 16^{\prime} 48^{\prime \prime}$ $\mathrm{N}$ y $72^{\circ} 02$ ' $21^{\prime \prime} \mathrm{W}$; y limita al occidente con Puerto Bolívar, el cual opera para el transporte directo de carbón para la empresa Carbones del Cerrejón Limited (CORPOGUAJIRA, 2006).

Desde el punto de vista biótico, el área está incluida en la unidad biogeográfica del cinturón árido pericaribeño en la provincia Alta Guajira, que presenta condiciones especiales que la clasifican como un desierto con vegetación de matorral desértico subtropical (Hernández-Camacho, Hurtado-Guerra, OrtizQuijano, \& Walschburger, 1992). La costa está bordeada en su mayor parte por formaciones densas del mangle rojo (Rhizophora mangle) y mangle negro (Avicennia germinans), de importancia a nivel nacional por considerarse especies en estado de amenaza (Sánchez-Páez et al., 2000; Gil-Torres et al., 2009). El promedio mensual de temperatura fluctúa entre $29.8{ }^{\circ} \mathrm{C}$ (octubre) y $25.4{ }^{\circ} \mathrm{C}$ (enero), y el de la salinidad superficial entre $36.5 \%$ (agosto) y $34.4 \%$ (diciembre) (Solano, 1994). La oferta pluviométrica a lo largo de cada año es irregular, se presentan dos temporadas, la primera alrededor de abril o mayo, correspondiente con el período de menor pluviosidad, y la segunda en septiembre, octubre y noviembre, siendo éste el de mayor intensidad y a veces el único período lluvioso perceptible en el año (Instituto Geográfico Agustín Codazzi, 2014; Gil-Torres et al., 2009). El complejo de ecosistemas de manglares, herbazales, arbustales, las praderas de fanerógamas, corales y fondos lodosos, son de importancia de conservación (Gutiérrez-Moreno, Alonso, \& Segura-Quintero, 2008; Galindo, Marcelo, Bernal, Vergara, \& Betancourth, 2009). Razón por la cual actualmente Bahía Portete es un área protegida de tipo marino costera, que se encuentra dentro 


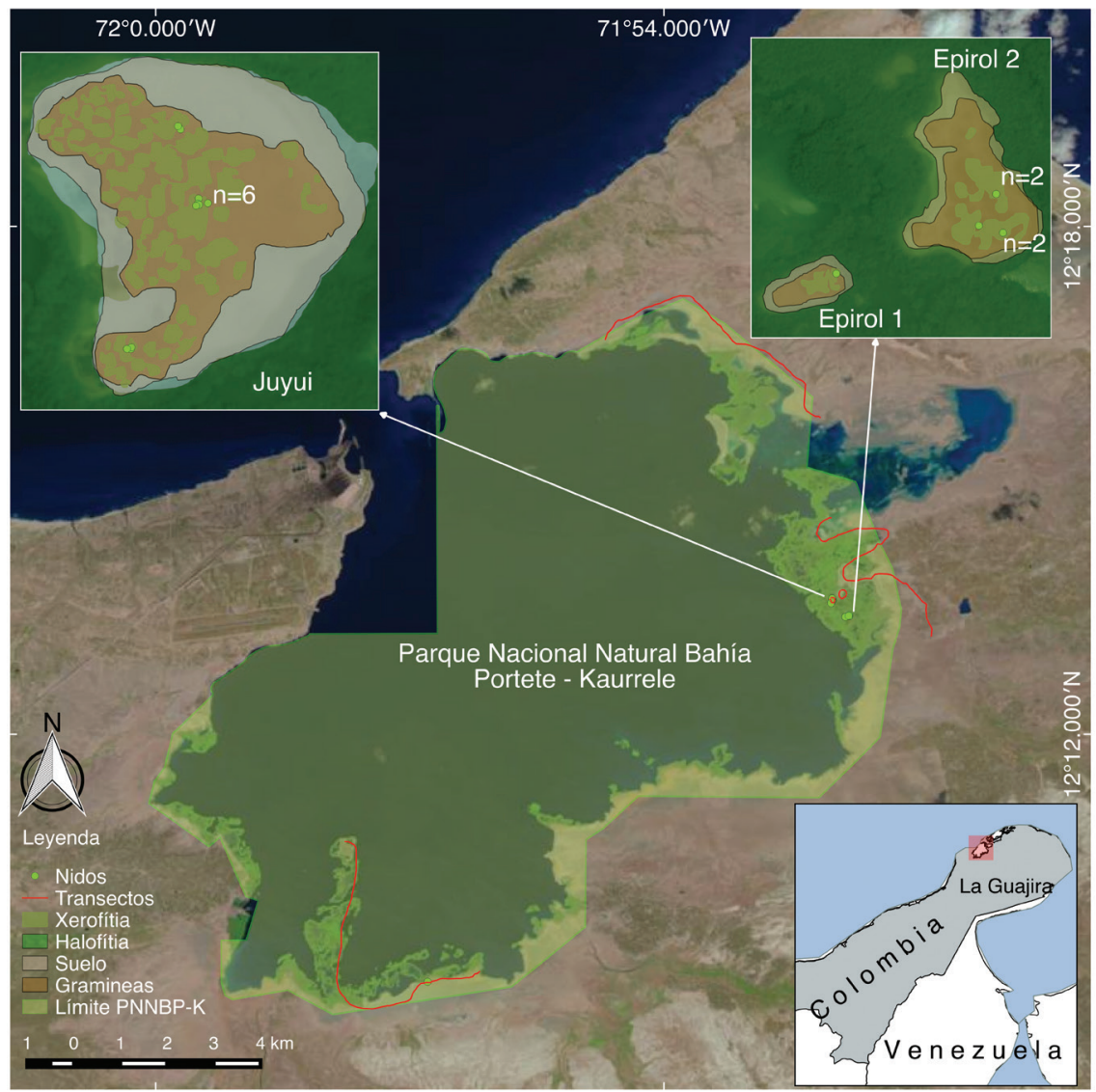

Fig. 1. Bahía Portete. En la ampliación las áreas de importancia para la anidación, n: corresponde al número de nidos hallados para 2008 en los lugares de anidación colonial.

Fig. 1. Bahía Portete. In expanding areas of importance for nesting, n: correspond to the number of nests found in 2008 in colonial nesting sites.

de sistema de Parques Nacionales Naturales de Colombia (Ministerio de Ambiente y Desarrollo Sostenible, 2014).

Ecología de la anidación: Se realizó una búsqueda intensiva de nidos en la bahía durante julio 2007, agosto 2008, junio a agosto 2009, y de marzo a agosto 2010 y 2011. Para ello, se efectuaron transectos diurnos entre las 7:00 y las 15:00 h. Los desplazamientos se realizaron en bote o caminando de acuerdo con la accesibilidad del terreno para el registro de los rastros de arribo de hembras grávidas, y los indicios de tapado de las posturas, para definir la ubicación de las nidadas.
Con la ubicación del nido, se registró su georreferenciación con el uso de un GPS (Garmin 60CSx); seguidamente se buscó la abertura del nido y se determinó su orientación cardinal empleando una brújula. En la parte externa del nido, se midió la distancia a la orilla del espejo de agua empleando una cinta métrica de $50 \mathrm{~m}$, y la distancia del nido a la especie vegetal más cercana. Se realizó la medición de las variables morfométricas del nido tales como: ancho de la boca, profundidad al primer huevo y profundidad total de la cámara. Se tomaron muestras de suelo de los lugares donde se hallaron los nidos para determinar las características del suelo de las nidadas, las cuales fueron analizadas 
en el laboratorio del Instituto Colombiano Agropecuario (ICA) para el análisis granulométrico con la prueba del higrómetro de Bouyoucos (Medina García \& Núñez, 2007). Se determinó además el número de huevos en la cámara, y se evitó alterar el polo de desarrollo embrionario marcándolos en la parte superior (Ferguson, 1985; Hutton \& Webb, 1992; Bolton, 1994), y se anotó el orden de recolecta. La viabilidad de los huevos se determinó evaluando la banda de desarrollo embrionario, clasificándolos de la siguiente manera: Fertilizados (F), si presentaban la banda opaca sin alteraciones; Muerte Embrionaria (M), cuando la banda opaca mostraba alteraciones en sus límites o lagunas en su interior o el huevo se encontraba en estado de descomposición; Huevos no fertilizados (I), cuando no mostraban la banda opaca o la formación del embrión, o a la luz se observan traslúcidos o amarillentos (Ferguson, 1985). En cada uno de los huevos se registró el diámetro mayor y menor empleando un calibrador digital tipo vernier (Electronic Digital Cliper $\pm 0.1 \mathrm{~mm}$ ) y el peso con una balanza digital (Camry $\pm 0.1 \mathrm{~g}$ ). Posteriormente, los huevos fueron reubicados en el nido en el orden descendente de recolecta. Desde 2008, los nidos fueron objeto de seguimiento para relacionar el tamaño de las crías al momento de nacer con el tamaño del huevo, para ello, se estimó el tiempo de nacimiento de acuerdo a la banda de desarrollo embrionario para posteriores revisiones a los 70, 75 y 80 días. Se empleó como indicador de acercamiento a la fecha de eclosión, la emisión de sonidos agudos dentro del huevo y la aparición de la marca de rotura de la cáscara realizada por el embrión que usa su "diente de huevo". Al instante del nacimiento, en los animales se anotó el correspondiente número del huevo y se registró la longitud total, la longitud rostro cloaca empleando una cinta métrica y el peso con una balanza digital (Camry $\pm 0.1 \mathrm{~g}$ ); de los análisis, se excluyó la información de los huevos que incluyeron nacimientos de individuos mal formados $(n=126$; Ferguson, 1985; Huchzermeyer, 2003).
Cronología de anidamiento: Adicionalmente a los transectos realizados para la búsqueda de nidos y el seguimiento del desarrollo de las nidadas, se hizo el registro mensual de la frecuencia de los rastros de llegada de las hembras grávidas a las áreas de anidación; también se registró la fecha de elaboración del nido, la eclosión y la aparición de crías en el manglar para plantear la cronología de eventos de anidación en Bahía Portete. Para identificar cuál variable ambiental se relacionó con el comportamiento reproductivo de C. acutus en el área, se instaló en 2010 una estación meteorológica portátil (PCE-FWS 20) a 1 km del área de anidación de mayor presencia de nidos.

Análisis de información: Con la frecuencia del número de nidos por área, para cada año se determinó las áreas de importancia para la anidación. Esta información también se empleó para estimar la densidad de nidadas en estas mismas áreas, empleando el programa de información geográfica QGIS 2.10.

Por otro lado, se realizó un análisis de varianza para determinar diferencias entre los años con las variables evaluadas en los nidos, la orientación de la excavación y el registro de la biometría de los huevos. Así mismo, se evaluó la variación temporal de la viabilidad de los huevos categorizándolos en fértiles y no fértiles. Para determinar estas diferencias entre años, previamente se evaluaron en cada variable los supuestos de homogeneidad de las varianzas y la normalidad de los residuos de cada variable. Las variables que cumplieron con los dos supuestos se sometieron a estadística paramétrica (Fisher, F) y las que no, a la estadística no paramétrica de Kruskal Walis (KW). Para esto, se empleó el paquete estadístico R (R Development Core Team, 2011), con la ayuda de la interfaz Rcomander (Fox, 2005) y el paquete gráfico ggplot2 (Wickham, 2009).

Para determinar si el tamaño del huevo (diámetro mayor) está relacionado con la longitud total de la cría al instante de nacer, se realizó una regresión lineal entre ambas variables. Igualmente se empleó este análisis para determinar si la longitud total del animal influye 
como variable predictora del peso del animal al instante del nacimiento, con previa transformación de los datos a logaritmo base 10 .

Con la información registrada en la cronología de anidamiento y de la estación climática, se propuso un modelo del comportamiento reproductivo de C. acutus en Bahía Portete. Los registros de los datos ambientales fueron asociados con la frecuencia de los eventos reproductivos para determinar cuál podría ser la variable que desencadena el comportamiento reproductivo en el área.

\section{RESULTADOS}

Ecología de la anidación: La búsqueda de nidos se realizó en todo el litoral, correspondiente a $55.12 \mathrm{~km}$ de longitud, con un total de 241 horas de esfuerzo en la bahía y el interior del manglar en Bahía Portete. Se determinaron cuatro áreas importantes para la anidación: las islas de Juyui $\left(12^{\circ} 13^{\prime} 35.22^{\prime \prime} \mathrm{N}\right.$ $\left.71^{\circ} 52^{\prime} 0.33^{\prime \prime} \mathrm{W}\right)$, de Epirol 1 (12 $13^{\circ}$ '24.24” N 71'51'48.78” W), de Epirol 2 (12 13 '24.18” N - 71 $\left.{ }^{\circ} 51^{\prime} 48.28^{\prime \prime} \mathrm{W}\right)$, y las dunas de arena cercanas al $R$. mangle en la costa del sector de Puerto Portete (129'4.47' N - 71'56'47.48' W, Fig. 1). Estas islas se encuentran a $1 \mathrm{~km}$ de la costa y están rodeadas de manglar, con elevaciones mayores a un metro sobre el nivel del mar; al interior están colonizadas por vegetación xerofítica y presentan poca intervención humana.
Entre 2007 y 2011 se localizaron 84 nidos, de los cuales 71 fueron caracterizados. En 2011 se observó el mayor número de nidos, seguido por 2009 y 2010 con 31, 17 y 14 nidos, respectivamente (Cuadro 1). La Isla de Juyui presentó el mayor número de nidos durante todos los años de estudio (53 nidos), seguida por el sitio Epirol 2 (17 nidos). Ambos sitios coincidieron en presentar la mayor cobertura de manglar de Bahía Portete (Fig. 1). En total se localizaron siete sitios de anidación colonial, tres en la isla Juyui y dos tanto en Epirol 1, como en Epirol 2 (Fig. 2A). Cada colonia estuvo constituida de 2 a 6 nidos y la distancia promedio entre nidadas en estas zonas fueron de $42.97 \pm 10.66 \mathrm{~cm}$ ( $\mathrm{n}=$ 6). De acuerdo a la información del cuadro 1, la mayor densidad de nidos se presentó en el 2011, siendo mayor en Epirol 1 (0.0588 ha, 34.013 Nidos/ha, $n=2$ ), seguida de Juyui (1.0689 ha, $17.77 \mathrm{Nidos} / \mathrm{ha}, \mathrm{n}=19)$ y por último Epirol 2 (0.2819 ha, 17.73 Nidos/ha, $n=5)$. Durante todo el estudio, el número de huevos promedio por nido fue de $28.4 \pm 6.63$, y en 2007 se obtuvo el mayor promedio (Cuadro 1). La comparación entre años del número de huevos por nido no presentó diferencias (ANOVA $\left.\mathrm{F}_{3.43}=0.715638, \mathrm{p}>0.05\right)$.

Durante la inspección de los nidos en 2008, se observaron posturas yuxtapuestas en Puerto Portete con la presencia de cuatro nidos con huevos sin éxito de eclosión $(n=41,41$, 45, 15 huevos; Fig. 2B) que probablemente

CUADRO 1

Nidos localizados de Crocodylus acutus en Bahía Portete

TABLE 1

Crocodylus acutus nests located in Bahia Portete

\begin{tabular}{lccccc} 
Año & $\begin{array}{c}\text { Total nidos } \\
\text { localizados }\end{array}$ & $\begin{array}{c}\text { Número de nidos } \\
\text { por localidad }\end{array}$ & $\begin{array}{c}\text { Número de nidos } \\
\text { medidos }\end{array}$ & $\begin{array}{c}\text { Promedio } \\
\text { de huevos }\end{array}$ & $\begin{array}{c}\text { Intervalo de } \\
\text { huevos }\end{array}$ \\
\hline 2007 & 10 & $8^{\mathrm{J}}, 1^{\mathrm{E} 2}, 1^{\mathrm{P}}$ & 8 & $31 \pm 7.4$ & $20-42$ \\
2008 & 12 & $9^{\mathrm{J}}, 2^{\mathrm{E} 2}, 1^{\mathrm{P}}$ & 8 & $26.1 \pm 11.5$ & $16-41$ \\
2009 & 17 & $10^{\mathrm{J}}, 1^{\mathrm{E} 1}, 5^{\mathrm{E} 2}, 1^{\mathrm{P}}$ & 17 & $29.4 \pm 6.5$ & $18-44$ \\
2010 & 14 & $7^{\mathrm{J}}, 2^{\mathrm{E} 1}, 4^{\mathrm{E} 2}, 1^{\mathrm{P}}$ & 14 & $27.8 \pm 6.0$ & $17-36$ \\
2011 & 31 & $19^{\mathrm{J}}, 2^{\mathrm{E} 1}, 5^{\mathrm{E} 2}, 5^{\mathrm{P}}$ & 24 & $28.7 \pm 6.2$ & $20-46$ \\
Total & 84 & & 71 & $28.4 \pm 6.6$ & $16-46$ \\
\hline
\end{tabular}

J: Juyui, E1: Epirol 1, E2: Epirol 2 y P: Puerto Portete. 

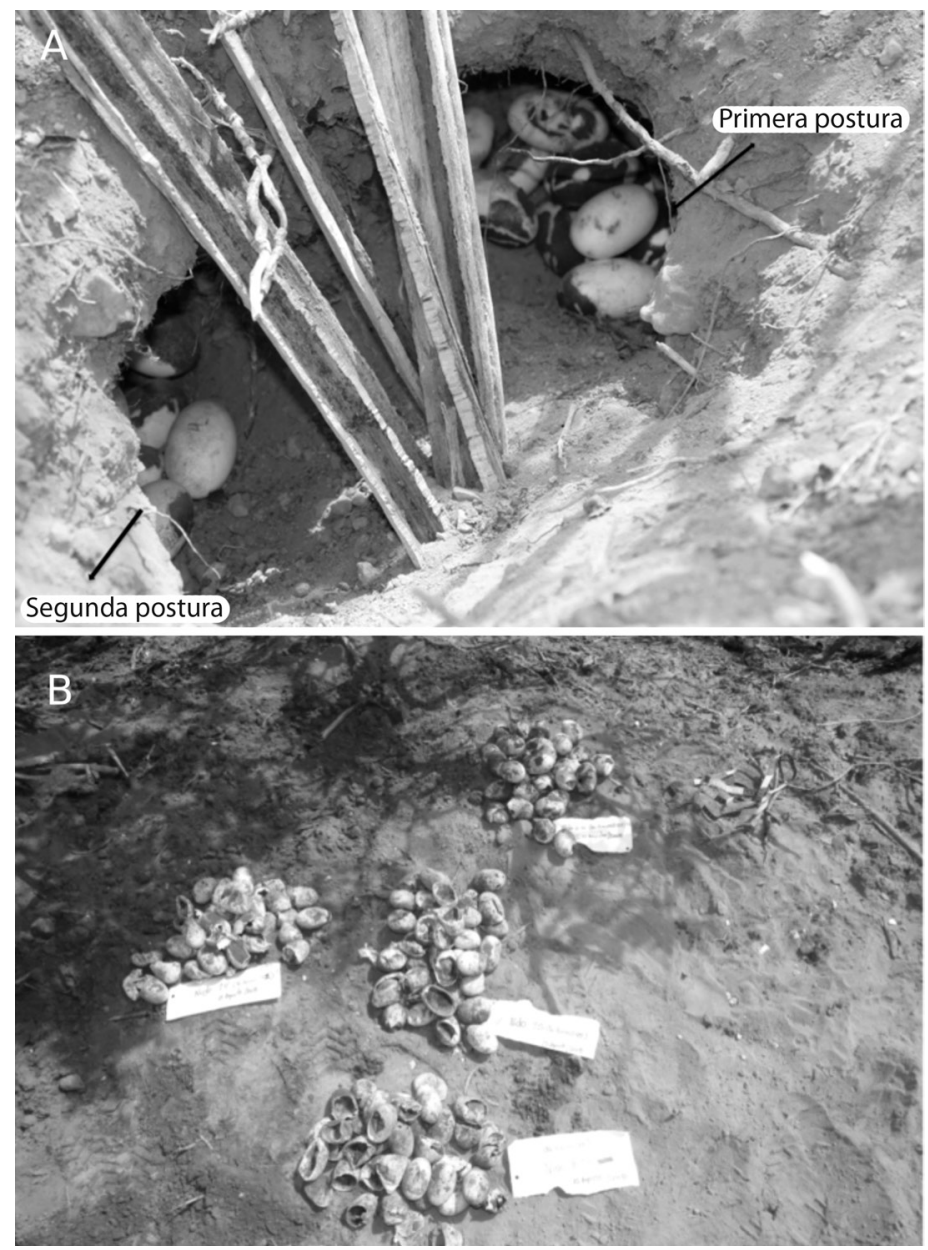

Fig. 2. A. Dos nidadas en la isla Epirol 2 en el sector donde se presenta anidación colonial. En la primera postura se dañaron seis huevos por la ruptura de la cáscara durante la elaboración del segundo nido. B. Localización de nidadas adyacentes a la nidada de Puerto Portete en 2008. Estas nidadas eran de años anteriores y los huevos presentaban al interior materia orgánica producto de la descomposición del embrión. Por un similar número de huevos puede tratarse de la misma hembra. El infructuoso éxito de eclosión pudo ser debido a los componentes del suelo.

Fig. 2. A. Two nests on the island Epirol 2 in the area where colonial nesting occurs. In the first position six eggs were damaged by the rupture of the shell during the elaboration of second nest. B. Location nests adjacent to nest of Puerto Portete in 2008. These nests were from previous years and had eggs into organic matter product of the embryo descomposition. By a similar number of eggs can it be treated is the same female. The unsuccessful hatching could be due to soil components.

correspondieron a puestas de años anteriores. Estos huevos se caracterizaron por presentar al interior materia orgánica embrionaria en descomposición. Es probable que esas posturas fueran de la misma hembra, debido a que la cantidad de huevos en buen estado $(n=40)$ fue similar al número de huevos infructuosos.

Con respecto a la orientación de los nidos, el $37 \%$ de los nidos estuvieron orientados hacia el norte y un $20 \%$ hacia el occidente (Cuadro 2). Sin embargo, no se registró diferencias significativas en las orientaciones de la oquedad (ANOVA de KW: $\mathrm{H}=7.81$, $\mathrm{p}>0.05$ ). La distancia entre los nidos y la orilla del espejo de agua marina fue muy variable entre nidos, con un promedio de $31 \pm 15.35 \mathrm{~m}$ (Intervalo $3-30, \mathrm{n}=$ 31, Cuadro 3). A pesar de eso, no varió de un año y otro (ANOVA de KW: $\mathrm{H}=1.03$, $\mathrm{p}>0.05$ ). 
CUADRO 2

Orientación de la abertura del nido de Crocodylus acutus

TABLE 2

Orientation of the nest opening of Crocodylus acutus

\begin{tabular}{cccccccccc} 
Año & Noroccidente & Norte & Occidente & Oriente & Sur & Nororiente & Suroccidente & Suroriente & Total \\
2008 & - & 2 & 3 & 2 & 1 & 2 & - & - & 10 \\
2009 & - & 7 & 2 & 3 & 5 & & - & - & 17 \\
2010 & 1 & 6 & 3 & 1 & 1 & & 1 & 1 & 14 \\
Total & 1 & 15 & 8 & 6 & 7 & 2 & 1 & 1 & 41 \\
\hline
\end{tabular}

Los nidos fueron elaborados a una distancia promedio de $50.9 \pm 39.60 \mathrm{~cm}$ de la vegetación más cercana. En un total de 43 nidos, el 48\% se construyeron en cercanía de Stenocereus griseus (Cactaceae) $(2008=8,2009=7,2010=$ 6 ), seguidos de un $32 \%$ cercano a Bauhinia purpurea (Caesalpiniaceae) $(2008=3,2009=$ 7, 2010=4). La profundidad hasta el primer huevo promedio de $26.43 \pm 9.05 \mathrm{~cm}$ y una profundidad total de la cámara promedio de $42.85 \pm 10.01 \mathrm{~cm}$.

Las características granulométricas del suelo observadas en los nidos en cada una de las áreas de anidación mostraron a la isla Juyui con el mayor porcentaje de arena. En el área de nidos individuales fue del $71.9 \% \mathrm{y}$ en los nidos coloniales fue del $71.44 \%$ (Fig. 3). Para el caso de Puerto Portete, fue el área con el mayor porcentaje de limo (37.27\%) y con los menores porcentajes de arena y arcilla, presentando el 51.69 y $11.04 \%$ de la composición, respectivamente.

Con respecto a la evaluación de la fertilidad de los huevos, se observaron en total 2085 huevos y sólo el $95 \%$ se encontraron fertilizados (Cuadro 3). Los huevos de C. acutus presentaron un diámetro mayor promedio de 71.84 $\pm 3.54 \mathrm{~mm}$ (Intervalo 52.83-97.15, Cuadro 3), siendo variable en cada año (ANOVA de KW= $44.8, \mathrm{p}<0.05)$. El peso promedio de los huevos fue de $81.54 \pm 9.99 \mathrm{~g}$ (Intervalo 43-104.1, Cuadro 3) y varió significativamente entre los años (ANOVA de $\mathrm{KW}$ : $\mathrm{H}=44.9$, $\mathrm{p}<0.05$ ), con 2011 el de mayor peso promedio registrado y la mayor biomasa total (53 737.2 g).

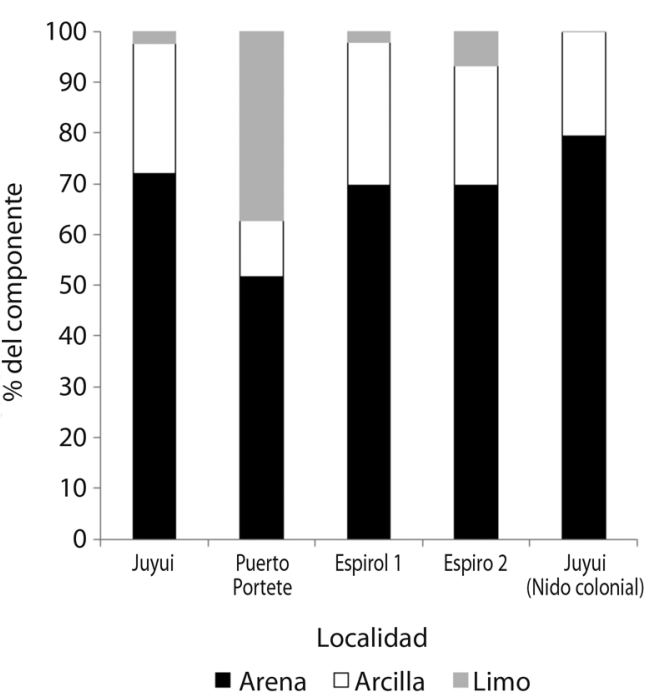

Fig. 3. Caracterización de los componentes del suelo en las áreas de anidación de Crocodylus acutus en Bahía Portete. Fig. 3. Characterization of soil components in nesting areas of Crocodylus acutus in Bahía Portete.

Se realizó la biometría de un total de 956 crías al instante del nacimiento, las cuales presentaron un promedio de longitud total de $25.47 \pm 1.16 \mathrm{~cm}$ (Intervalo $=20.10-27.8$, Cuadro 3). La comparación de la longitud total de la crías entre un año y otro varió significativamente (ANOVA de KW: $\mathrm{H}=91.03$; $\mathrm{p}<0.05)$. Las crías presentaron un peso promedio de $57.40 \pm 6.30 \mathrm{~g}($ Intervalo= 40.7-72.5), pero difirieron entre años (ANOVA de KW: $\mathrm{H}=14.8568, \mathrm{p}<0.05)$. El análisis de regresión lineal entre el tamaño del huevo y el tamaño de la cría al instante del nacimiento fue positiva 


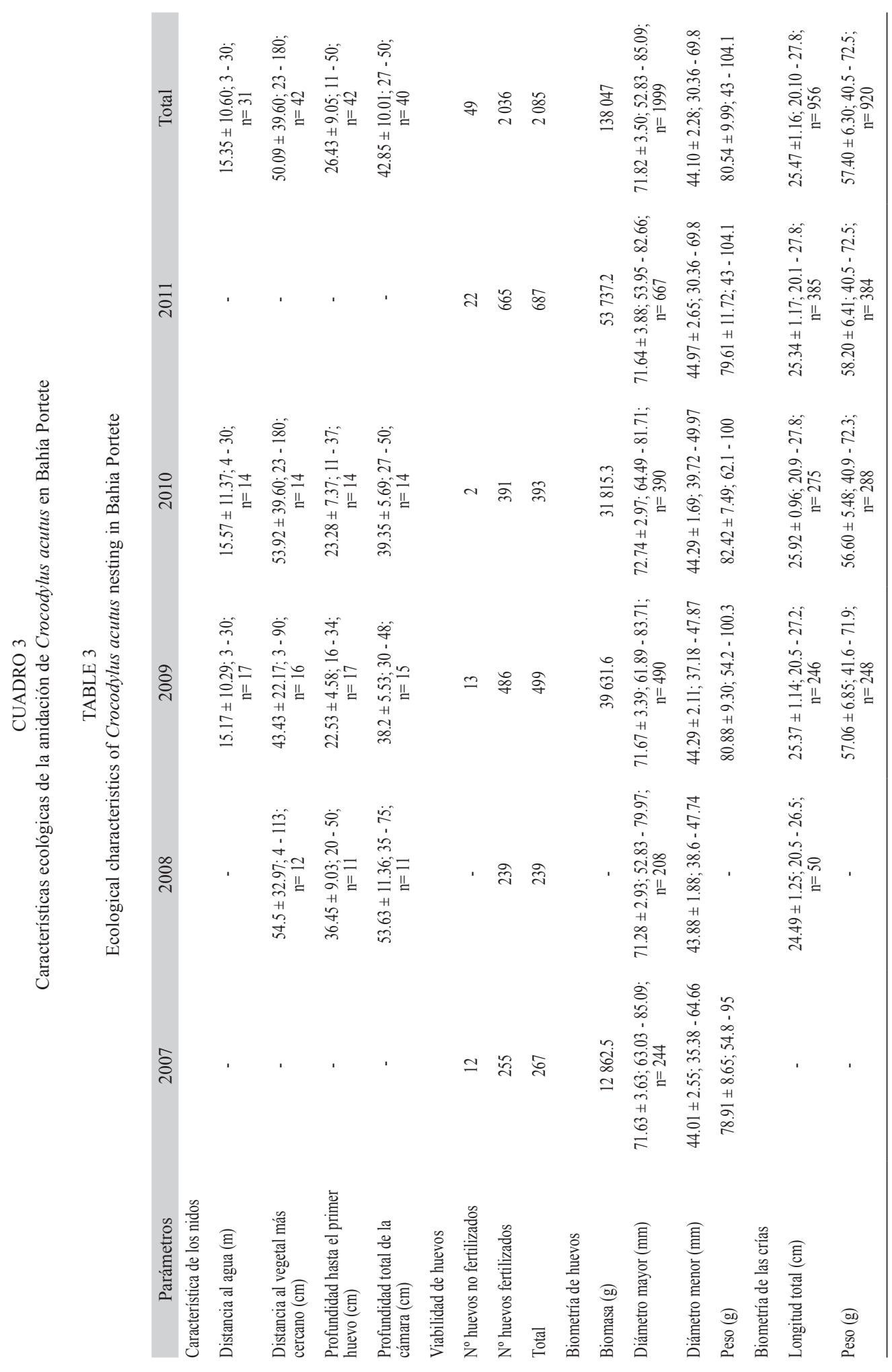


y significativa, pero con un baja dependencia $\left(\mathrm{R}^{2}=0.12, \mathrm{~F}=129.1, \mathrm{p}<0.05\right.$, Fig. 4A). Para el caso de la relación entre la longitud de la cría al instante del nacimiento y el peso, fue positiva y significativa, también con una menor dependencia $\left(\mathrm{R}^{2}=0.31, \mathrm{~F}=392.3, \mathrm{p}<0.05\right.$, Fig. 4B).
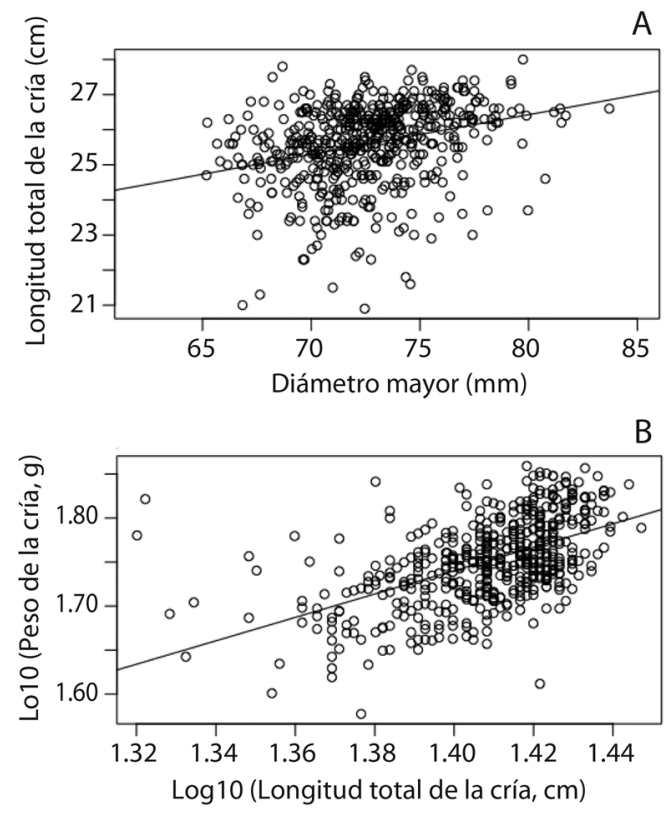

Fig. 4. A. Correlación entre el diámetro mayor del huevo y la longitud total de las crías al instante de nacer (modelo Longitud Total de la Cría $=15.97765+0.13105 *$ Diámetro Mayor), B. Correlación entre la longitud total de las crías y el peso de la cría al instante de nacer (modelo Log10(Peso) $=-0.37999+1.36610 * \log 10$ (Longitud Total de la Cría).

Fig. 4. A. Correlation between the larger diameter of the egg and the total length of the hatchlings birth instantly (model Length Total Hatchling $=15.97765+0.13105$ * Larger Diameter), B. Correlation between total length and weight of hatchlings birth instantly ( $\log 10$ model $($ Weigh $)=-0.37999+1.36610 * \log 10$ (Total Length of the Hatchling).

Cronología del anidamiento: Se determinó que $C$. acutus realiza los arribos para anidamiento a finales de mayo; entre mayo y la primera semana de julio se realiza la anidación; y para mediados de agosto las eclosiones (Fig. 5). El seguimiento del tiempo de incubación durante los cuatro años, presentó un promedio de duración de $76.1 \pm 7.2$ días (Intervalo
62-89). En general, el período reproductivo para Bahía Portete inicia en marzo con el cortejo y la cópula, evento que coincide con el primer período lluvioso que se presenta en el año, y las eclosiones a mediados de agosto, época correspondiente al segundo período lluvioso del año y el cual tiene la mayor probabilidad de ocurrencia en el año (Instituto Geográfico Agustín Codazzi, 2014) (Fig. 6).

\section{DISCUSIÓN}

Luego de más de una década, se describe la ecología y cronología del proceso de anidación de $C$. acutus en Bahía Portete. Ubicada al extremo norte de Colombia, se identificaron cuatro áreas de anidación. Esto despierta un interés para desarrollar acciones de conservación, debido a que son áreas poco visitadas por los indígenas Wayuü que se dedican a la pesca, están protegidas por una barrera de manglar alrededor de ellas y están ubicadas a una altura mayor a un metro sobre el nivel del mar. Estas áreas fueron similares a las descripciones de Mazzotti (1989) para la población de la Florida y de Rodríguez-Soberon, Tabet, y Berovides (2002) para Cuba.

En las tres islas se observó la anidación colonial, la cual ha sido documentada en otros países con presencia de C. acutus (Thorbjarnarson, 1989; Rodríguez-Soberon, Tabet, \& Berovides, 2002; Balaguera-Reina, VenegasAnaya, Sanjur, Lessios, \& Densmore III, 2015). Este tipo de comportamiento genera posturas yuxtapuestas y se convierte en un factor desfavorable para el éxito de la población de $C$. acutus en Bahía Portete. La distancia promedio entre nidadas coloniales observada fue menor $(<0.5 \mathrm{~m})$ a las observadas en otros países. Por ejemplo, en Florida es de $1 \mathrm{~m}$ (Kushlan \& Mazzotti, 1989), en México de 2 a 15 m (Sigler, 2010), en Cuba de 10 m (Rodríguez-Soberon, 2000) y en Panamá de $6.2 \mathrm{~m}$ (Balaguera-Reina et al., 2015). Sin duda, la anidación colonial puede ser una de las causas de la pérdida de cuatro nidadas observadas en 2008, y sugiere que la disponibilidad y calidad de sitios para anidación en Bahía Portete son escasos. 

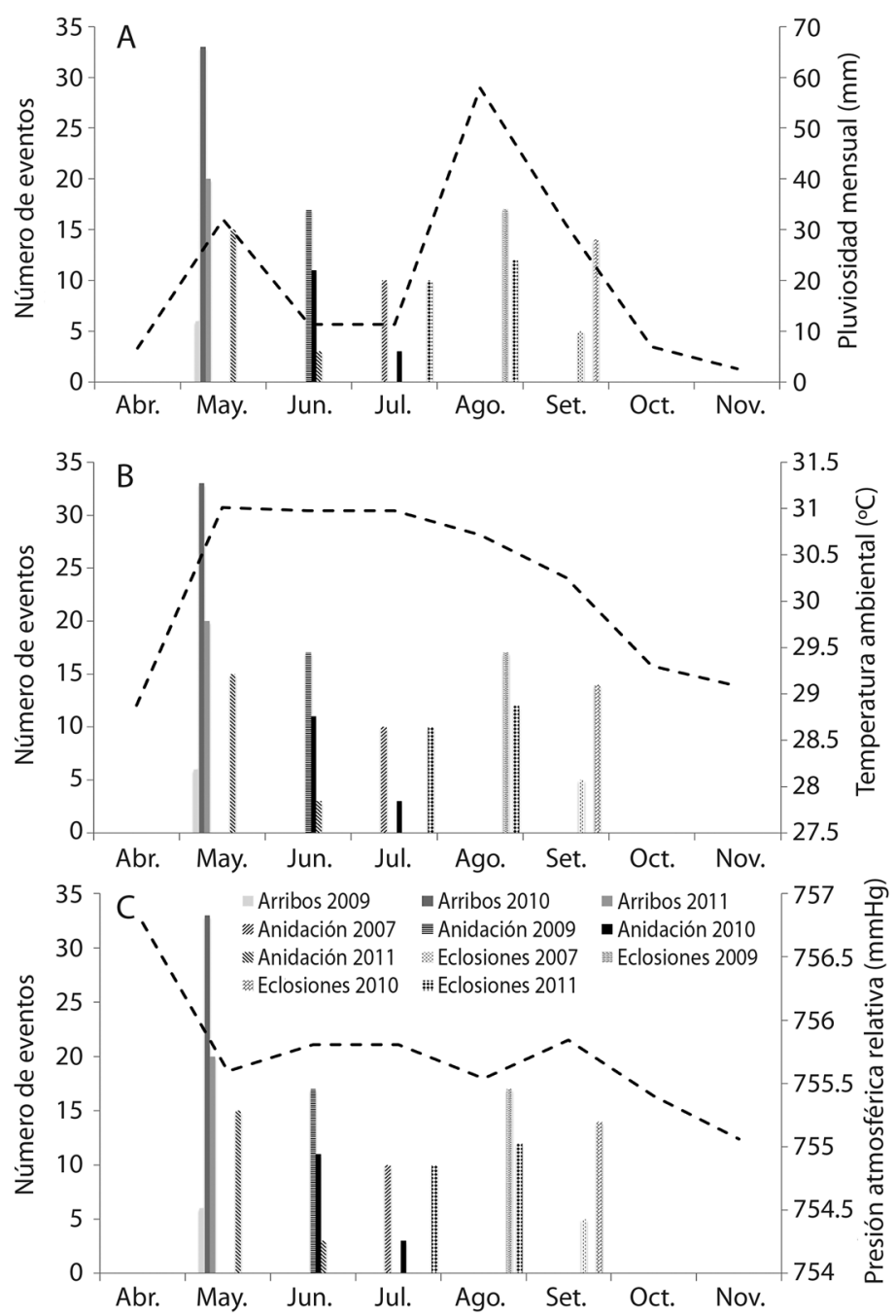

Fig. 5. Registros de actividades de anidamiento de Crocodylus acutus en Bahía Portete para cada una de variables ambientales registradas durante el 2010 con una estación meteorológica ubicada a una distancia de $1 \mathrm{~km}$ del área de anidación Juyui, A. Pluviosidad mensual, B. Temperatura y C. Presión atmosférica.

Fig. 5. Records of activities Crocodylus acutus nesting in Bahia Portete for each of environmental variables registered during 2010 with a weather station located at a distance of $1 \mathrm{~km}$ from nesting area Juyui, A. Monthly Rainfall, B. Temperature and C. Atmospheric pressure.

A lo anterior se le suman otros factores que no permiten el éxito de eclosión de $C$. acutus en Bahía Portete. Entre estos se detectó la influencia de los cambios drásticos de temperatura que puede producir la desecación y desnaturalización de la proteína del albumen, con la consecuente mortalidad de huevos. Otro factor, fue la alta humedad proporcionada por las lluvias durante el segundo período lluvioso del año,

que puede generar un proceso de hinchamiento de los huevos por afectar el intercambio gaseoso, un fenómeno que también afecta la anidación de otras especies de crocodílidos (Lutz \& Dunbar-Cooper, 1984; Grigg, 1987; Hutton \& Webb, 1992). El análisis de suelo también permitió identificar otro posible factor que influyó en el éxito de eclosión de los huevos. El alto porcentaje de limo en el suelo de los nidos 


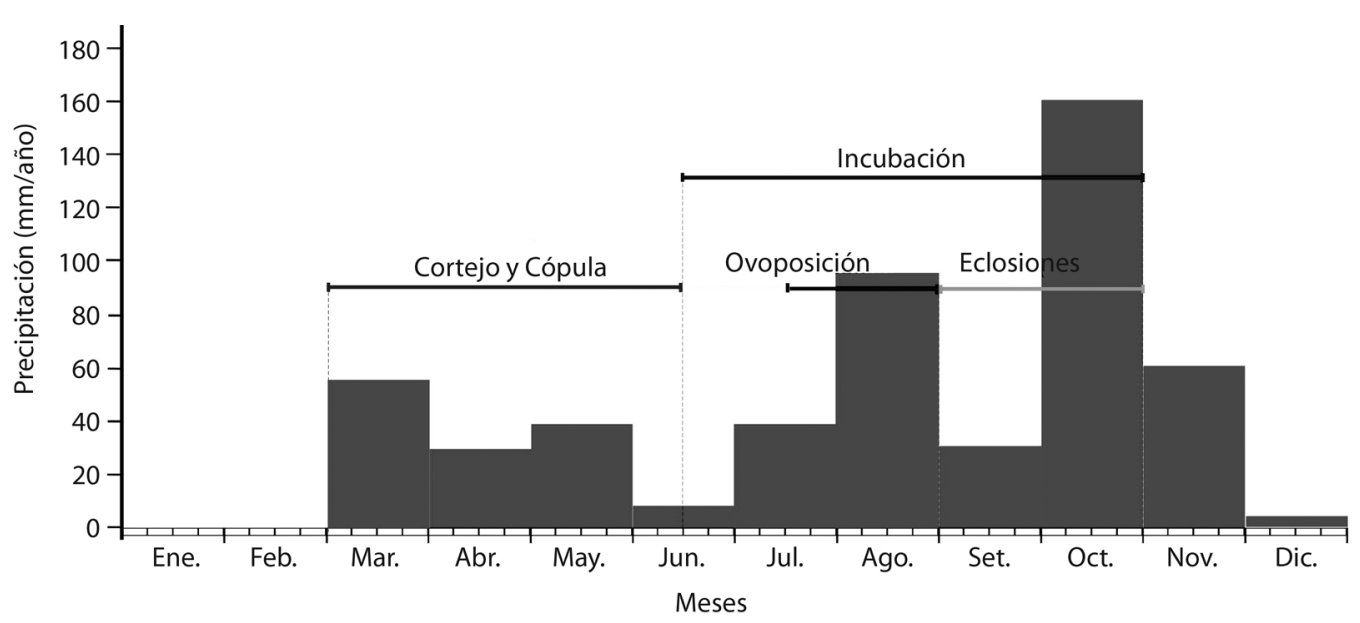

Fig. 6. Desarrollo del comportamiento reproductivo de Crocodylus acutus en Bahía Portete.

Fig. 6. Development of reproductive behavior of Crocodylus acutus in Bahía Portete.

observados en Puerto Portete, junto con la acción climática, pudo afectar la respiración de los huevos (Casas-Andreu, 2003) y por lo tanto, convertirse en otra causa del fracaso de eclosión de los cuatro nidos observados. Finalmente, se tiene la posible depredación por Procyon cancrivorus o Conepatus semistriatus, las cuales fueron observadas en el área, y de las que se tienen antecedentes de depredación de nidadas de C. acutus (Medem, 1981; Mazzotti, 1999). Lo anterior sugiere la necesidad de plantear una estrategia de manejo de las nidadas en riesgo para lograr mantener el tamaño de la población a una condición favorable en Bahía Portete.

La comparación del tamaño de la nidada de C. acutus en Bahía Portete con otras localidades, resultó ser mayor que en áreas pantanosas de México $(26.0 \pm 7.48$ huevos, Intervalo= 16-34, $\mathrm{n}=4$; Cupul-Magaña, De Niz-Villaseñor, Reyes-Juárez, \& Rubio-Delgado, 2004), en Cuba (24.8, Intervalo $=8-51, \mathrm{n}=720$; Rodríguez-Soberon et al., 2002), en Jamaica (22.5 \pm 2.7, $\mathrm{n}=14$; Thorbjarnarson, 1988), República Dominicana (21.6 \pm 6.4 , Intervalo= 9-6, $n=62$; Schurbert, 2002), Belize (22.3 \pm 6 , Intervalo $=12-32, \mathrm{n}=14$; Platt $\&$ Thorbjarnarson, 2000) y en Perú $(25 \pm 2, n=3$; Pérez \& Escobedo-Galván, 2005b). No obstante, resultó similar a las presentadas en otras localidades de
Colombia: Dibulla (de 23 a 48 huevos; Gómez \& Patiño, 2010) y Bahía de Cispatá (promedio 25.02 a 30.46; Sánchez-Páez, Ulloa-Delgado, \& Arsenio-Tavera, 2004; Ulloa-Delgado \& Sierra-Díaz, 2006; CVS, 2012), y menor a otra localidad en México (33.07 \pm 13.95 , Intervalo= 1-54, n=14; Casas-Andreu, 2003), y de Colombia, principalmente en Isla Fuerte donde se observaron de 18 a 20 huevos (Medem, 1981).

Los nidos de C. acutus en Bahía Portete son oquedades y por lo tanto las hembras no realizan montículos que sobrepasen el nivel del suelo como ocurre en otras localidades (Mazzotti, 1989; Charruau, 2012). El hecho de establecerse en cercanías a la vegetación xerofítica favorece la disminución de la incidencia directa de la radiación solar y a su vez facilita en parte la persistencia de la humedad durante el período de sequía. Observaciones de campo permitieron identificar que la niebla se condensa en las estructuras de Stenocereus griseus y desciende hasta las raíces, proporcionando parte de la humedad a los nidos. Esta apreciación merece un estudio para cuantificar el aporte de humedad por medio de las plantas xerofíticas. Este comportamiento es contrastante al observado en otro tipo de hábitat, donde los nidos son construidos distantes a la vegetación arbórea (CasasAndreu, 2003). Sin embargo, las características 
de los nidos de C. acutus en Bahía Portete se encuentran dentro de los parámetros registrados en otros lugares (Thorbjarnarson, 1989; Casas-Andreu, 2003; Cupul-Magaña et al., 2004; Hernández-Hurtado, Romero-Villaruel, \& Hernández-Hurtado, 2011).

Los nidos hallados tuvieron una tasa de fertilidad de huevos de más del $95 \%$, mayor a la reportada para México (promedio $80.7 \pm$ $21.0 \%$; Casas-Andreu, 2003) y la Florida (82.4 $\%$; Mazzotti, 1989). Esta tasa se puede considerar apropiada para el desarrollo de un programa de manejo de nidos al emplear una incubación de poca tecnología que propenda por una recuperación y estabilización de la población. Esto también puede propiciar un aprovechamiento con un enfoque precautorio de C. acutus en Bahía Portete, debido a que en 2011 se observaron 31 nidos, lo que sugiere una población de 31 hembras en estado reproductivo. La estrategia de recolecta e incubación de huevos de crocodílidos ha sido una herramienta empleada para el aumento de la población en otros lugares (Barros, Jiménez-Oraá, Heredia, \& Seijas, 2010; Espinosa-Blanco, Seijas, \& Hernández, 2013; Hernández-Hurtado, Vega-Villasante, Hernández-Hurtado, Cupul-Magaña, \& García de Quevedo-Machain, 2013).

El diámetro de los huevos de la población de $C$. acutus en Bahía Portete fue menor en comparación a las observadas en otras poblaciones, incluso a las más cercanas, como las del plano inundable de los ríos Jerez y Tapia. Están ubicada a $185 \mathrm{~km}$ hacia el sur de Bahía Portete y asociadas a zonas de manglar de pantanos de agua dulce, con influencia del mar durante la apertura de las desembocaduras de los ríos (Gómez \& Patiño, 2010). En esas poblaciones, Gómez y Patiño (2010) observaron huevos con un diámetro promedio de $79.86 \pm 2.94 \mathrm{~mm}$, que superan a los de Bahía Portete (ANOVA de KW: $\mathrm{H}=474.484 ; \mathrm{p}<0.05)$. Lo mismo ocurrió con el peso de los huevos, que tuvieron un valor promedio de $106.18 \pm 7.97 \mathrm{~g}$ (ANOVA de $\mathrm{KW}: \mathrm{H}=472.338 ; \mathrm{p}<0.05)$. Esta diferencia en diámetro y peso de los huevos, está poniendo en evidencia la diferencia en el tipo y calidad del hábitat que pueden estar experimentando las hembras de ambas áreas. Por ejemplo, las hembras de los ríos Jerez y Tapia alcanzan mayores tallas y en consecuencia, una mayor relación con el tamaño de la nidada y de los huevos (Gómez \& Patiño, 2010), que es típico de los cocodrilos (Greer, 1975; Thorbjarnarson, 1996). En contraste, tenemos las de Bahía Portete que interactúan con el ambiente marino y en consecuencia, puede estar influyendo en el tamaño y peso de los huevos. Esto puede ser concordante con lo documentado, puesto que $C$. acutus presenta menores tasas de crecimiento y alta tasa de mortalidad en las crías cuando habita ambientes salinos (Dunson, 1982; Dunson \& Mazzotii, 1989; Kushlan \& Mazzotti, 1989; Thorbjarnarson, 1989; Richards, Mooij, \& De Angelis, 2004; Hernández-Hurtado et al., 2006; Seijas, 2011). A pesar de lo anterior, no afecta la población adulta (Richards et al., 2004).

Por otro lado, en la población de C. acutus de Bahía Portete se observó una baja relación entre la longitud de la cría y el peso al nacimiento. Es probable que esto se deba a la deshidratación de los huevos durante la incubación producto a las elevadas temperaturas en el área. Durante la incubación, la cáscara del huevo se debilita y en algunos casos se quiebra, quedando expuesta la membrana a la influencia directa de las elevadas temperaturas en el desarrollo, con una pérdida de humedad y desecación del huevo. En consecuencia, la deshidratación influye en el tamaño de las crías al instante de nacer, o en el caso extremo ocasiona falta de absorción del vitelo y una muerte embrionaria (Mazzotii, 1983).

Con respecto a los tamaños de las crías al nacer, en la población de Bahía Portete presentaron una longitud total dentro de los valores documentados para C. acutus en otros países (Florida: $26.5 \pm 1.9 \mathrm{~cm}$; Brandt et al., 1995; río Tumbes, Perú: $24.54 \pm 0.98 \mathrm{~cm}$; Pérez \& Escobedo-Galván, 2005a; México: 24.0-27.0 cm; Álvarez del Toro, 2001; Jalisco, México: $26.32 \pm 0.50 \mathrm{~cm}$; Cupul-Magaña et al., 2004; Chiapas, México: $28.2 \pm 0.97 \mathrm{~cm}$; Sigler, 2010). Igualmente se observó para el peso (Florida, USA: $59.4 \pm 9.9 \mathrm{~g}$; Brandt et al., 1995; río Tumbes, Perú: $46.13 \pm 4.28$ g; 
Pérez \& Escobedo-Galván, 2005a; Jalisco, México: $60.44 \pm 2.37 \mathrm{~g}$; Cupul-Magaña et al., 2004; Chiapas, México: $69 \pm 9.7$ g; Sigler, 2010). Sólo superó a los documentados para Haití (Thorbjarnarson, 1996) y se debe al hábitat hipersalino que influye en el tamaño y tasa de crecimiento de los animales.

Para el caso del tiempo de incubación, en Bahía Portete C. acutus presentó menores valores a los observados en otros lugares (Florida: 85 días, Brandt et al., 1995; México: 80-83 días, Álvarez del Toro, 2001, Casas-Andreu, 2003; Jamaica: 84 días, Thorbjarnarson, 1989; Panamá: 80-90 días, Balaguera-Reina et al., 2015). El menor tiempo de incubación es evidencia de la influencia de las altas temperaturas y poca humedad que se presenta en el ecosistema subxerofítico de Bahía Portete, que consecuentemente afecta el microclima de los nidos.

En lo que respecta al período reproductivo de C. acutus en Bahía Portete, se observó que estuvo sincronizado con el desarrollo del período de lluvias, cuando ésta inició desde el primer semestre del año (a partir de marzo). Sin embargo, en los años en que no llovió durante el primer semestre, el posible factor desencadenante del inicio del comportamiento reproductivo pudo ser la percepción de la especie al cambio de la presión atmosférica o de la luminosidad, los cuales son imperceptibles para el humano. Sería importante que en estudios futuros se evalúe esta apreciación. Con respecto a las otras etapas del ciclo reproductivo, se observó que la ovoposición inicia de mayo a junio; la incubación entre junio e inicios de agosto; y las eclosiones a mediados y finales de agosto, que coincidió con el inicio del segundo y mayor período de lluvias en Bahía Portete. Esto último garantiza menores niveles de salinidad para favorecer un mejor desarrollo y supervivencia de las crías (Mazzotti, 1983; Mazzotti, Bohnsack, McMahon, \& Roos, 1986). Con lo anterior, estas etapas sólo coinciden con dos poblaciones: una ubicada en otra zona árida del Estado de Falcón en Venezuela y probablemente se deba a la similitud en la latitud y a las condiciones climáticas (Medem, 1983; Thorbjarnarson, 1989; Seijas,
Urdaneta, \& Barros, 2008); la segunda está en la Reserva de la Biosfera Banco Chinchorro Quintana Roo en México (Charruau, Thorbjarnarson, \& Hénaut, 2010). A diferencia de lo anterior, hay poblaciones de Colombia que se diferencian cronológicamente en el ciclo. Por ejemplo, en los ríos Jerez y Tapia, C. acutus inicia los anidamientos con un mes de anterioridad a lo ocurrido en Bahía Portete (Gómez $\&$ Patiño, 2010). En el caso del departamento del Chocó, el período reproductivo inicia entre enero y febrero y en los departamentos del Atlántico y Córdoba de febrero a marzo (Medem, 1981; Ulloa-Delgado, Sierra-Díaz, \& Cavanzo, 2005).

Al analizar toda la información de la ecología de anidación de $C$. acutus en Bahía Portete, es evidente que se necesita ejecutar un programa de manejo y conservación para aumentar el tamaño de la población y así evitar su extinción. Este programa debe tener un énfasis en la vinculación participativa de la comunidad de indígena Wayuü para concertar cada estrategia, entre las cuales tenemos: i) manejo de nidadas en riesgo. Tener en cuenta la alta fertilidad de la población y el efecto adverso del suelo y el clima que disminuyen el éxito de eclosión, se puede implementar el manejo de la incubación protegida como uno de los ejes del programa de conservación. Esto maximizaría las probabilidades de natalidad y el aumento en el aporte del potencial reproductivo de la población. Este proceso debería incluir el traslado de nidos en riesgos hacia áreas donde se generen similares condiciones de suelo al de la isla Juyui. En caso de no contar con esas áreas, lograr con la ayuda de los indígenas Wayuü una estrategia de incubación artesanal que no requiera de energía externa distinta a la oferta ambiental, y que disminuya los efectos drásticos de las variables climáticas, principalmente la incidencia directa de la radiación solar (Webb, Manolis, \& Whitehead, 1987; Barros et al., 2010; Charruau, 2012). ii) Aumentar el área de anidación. Esto logrará minimizar las nidadas coloniales. Se debe llegar a un proceso de concertación con las comunidad indígena Wayuü para identificar y proponer nuevas áreas 
de anidación para C. acutus. Estas áreas deben tener características ambientales similares a las identificadas en este estudio, utilizando como referencia la de la isla Juyui. iii) Delimitar las áreas de pesca en Bahía Portete. Concertar con las comunidades de pescadores áreas intangibles a la pesca donde se distribuyen los ejemplares adultos y jóvenes para no causar las muertes incidentales que ocasionan los aparejos de pesca. iv) Educar a la comunidad Wayuü. Es la población humana que tiene el mayor contacto con $C$. acutus y es necesario que siempre se mantengan educados sobre los aspectos biológicos y ecológicos de la especie; y de su importancia de conservarla para evitar la extinción de la población de Bahía Portete (Towsend, 2003). v) Conservación del hábitat. Por ser las áreas de mayor cobertura de manglar donde se observaron la mayor cantidad de nidos, se sugiere evitar la tala de esta vegetación para lograr mayores garantías de protección de los nidos, de oferta de alimento y de la supervivencia de las crías.

Estrategias planteadas como las anteriores han sido eficientes y exitosas en el manejo de cocodrílidos (Thorbjarnarson, 2001), principalmente en Colombia (Ulloa-Delgado \& Sierra-Díaz 2012), Argentina (Larriera \& Imhof, 2004) y Venezuela (Espinosa-Blanco, Seijas, \& Hernández, 2010). Estas deben ser desarrolladas bajo la dirección de las entidades administradoras de los recursos naturales de Colombia, con la ayuda de los académicos y científicos y la participación del sector privado. Por último, es importante seguir realizando investigaciones permanentes sobre aspectos de la biología reproductiva, ecología trófica, genética de poblaciones, dinámica poblacional, reclutamiento y dispersión de $C$ acutus en Bahía Portete que permitan fortalecer los futuros programas de conservación y manejo.

\section{AGRADECIMIENTOS}

Los autores expresan sus más sinceros agradecimientos a la comunidad Wayuü de Granvía, Iian, Uraichiquiru y Puerto Portete que participaron durante la ejecución del trabajo de campo, especialmente a los clanes Uriana y Epinayu. El equipo de trabajo de la sección ambiental del Carbones de Cerrejón Limited, coordinado por Gabriel Bustos que otorgaron el permiso de uso de los datos de cuatro años de desarrollo del programa Conservación de Especies Amenazadas de Cerrejón, de la misma manera la colaboración en la lectura del manuscrito a Paola Escobar Ramos y Pablo Lagares y los valiosos aportes de los pares de la revista. Esta publicación forma parte del trabajo de grado de John Jairo Gómez para optar por al título de MSc. Ciencias Ambientales (Universidad del Atlántico).

\section{RESUMEN}

En Colombia, C. acutus se encuentra catalogada en peligro crítico. Durante julio 2007, agosto 2008, junio y agosto 2009 y entre marzo y agosto 2010 y 2011, la población en Bahía Portete fue estudiada para evaluar la anidación y obtener la información necesaria para el ajuste de un programa de manejo acorde con la biología de la especie. Este estudio asumió la vinculación de la comunidad indígena Wayuü para emplear el modelo de acuerdo de conservación. Se realizaron transectos en toda la costa de la bahía para la búsqueda de nidadas. En la determinación de las áreas de importancia para la anidación, se registraron las características biométricas de los nidos, la biometría de huevos y crías, la fertilidad de huevos y eventos reproductivos. Como resultado se recorrieron $55.12 \mathrm{~km}$, se determinaron cuatro zonas de importancia para la anidación, la de mayor importancia fue la isla Juyui. En todas las áreas de anidación, variables como la anidación colonial o gregaria y las variables ambientales fueron factores que afectaron el éxito de eclosión. El $37 \%$ de los nidos estuvieron orientados hacia el norte y el $48 \%$ fueron construidos en cercanía de Stenocereus griseus. La isla Juyui presentó los mayores porcentaje de arena $(71.9 \%, 71.44 \%)$. El promedio de huevos por nido fue $28.42 \pm 6.63$. Los huevos presentaron un diámetro mayor promedio de $71.84 \pm 3.54 \mathrm{~mm}$ y peso $81.54 \pm 9.99 \mathrm{~g}$. Las crías presentaron un promedio de longitud total de $25.47 \pm 1.16 \mathrm{~cm}$. El período reproductivo para este sector de Colombia, se inicia en marzo y culmina en agosto. Las pocas áreas de anidación y la fertilidad del 95 $\%$ sugieren la ejecución de un programa conservación para C. acutus en Bahía Portete.

Palabras clave: cocodrilos, Crocodylus acutus, Bahía Portete, anidación, reproducción.

\section{REFERENCIAS}

Abadia, G. (1995). Crocodylus acutus (Cuvier 1807) in Bahía Portete. CSG Newsletter, 4, 9. 
Abadia, G. (1996). Population dynamics and conservation strategies for Crocodylus acutus in Bahía Portete, Colombia. In CSG (Crocodile Specialist Group) (Eds.), Crocodiles. Proceedings of the 13th Working Meeting of the Crocodile Specialist Group (pp. 176-183). Gland, Switzerland: IUCN-The World Conservation Union.

Álvarez del Toro, M. (2001). Los Crocodylia de México (1 ${ }^{\mathrm{a}}$ Edición). México D. F., México: Jiménez Editores e Impresos S.A.

Balaguera-Reina, S. A., Venegas-Anaya, M., Sanjur, O. I., Lessios, H. A., \& Densmore III, L1. D. (2015). Reproductive Ecology and Hatchling Growth Rates of the American Crocodile (Crocodylus acutus) on Coiba Island, Panama. South American Journal of Herpetology, 10, 10-22

Barros, T., Jiménez-Oraá, M., Heredia, J. H., \& Seijas, A. E. (2010). Colecta de huevos de caimanes (Crocodylus acutus y $C$. intermedius) en Venezuela para fines de conservación. En R. De Oliveira-Miranda, J. Lessmann, A. Rodríguez-Ferraro, \& F. RojasSuárez (Eds.), Ciencia y conservación de especies amenazadas en Venezuela: Conservación basada en evidencias e intervenciones estratégicas (pp. 77-86). Caracas, Venezuela: Grupo Intenso.

Bolton, M. (1994). La explotación del cocodrilo en cautividad. Guía de Conservación 22, Organización de las Naciones Unidas para la Agricultura y la Alimentación, Roma. http://www.fao.org/docrep/006/T0226S/ T0226S00.HTM. 24/03/2011

Brandt, L. A., Mazzotti, F. J., Wilcox, J. R., Barker, P. D., Hasty, G. F., \& Wasilewski, J. (1995). Status of the American crocodile (Crocodylus acutus) at a power plant site in Florida, USA. Herpetological Natural History, 3, 29-36.

Carbones del Cerrejón Limited. (2007). Diagnóstico poblacional y algunos aspectos bioecológicos del Kayuüshi (Crocodylus acutus cuvier, 1807) en Bahía Portete, península de La Guajira - Colombia (Informe). Barranquilla: Fundación Hidrobiológica George Dahl.

Carbones del Cerrejón Limited. (2008). Programa de conservación del Kayuüshi (Crocodylus acutus) y tortugas marinas en Bahía Portete, península de La Guajira-Colombia. Fase II (Informe). Barranquilla: Fundación Hidrobiológica George Dahl.

Carbones del Cerrejón Limited. (2009). Programa de conservación del Kayuüshi (Crocodylus acutus) y tortugas marinas en la Alta Guajira-Colombia (Informe). Barranquilla: Fundación Hidrobiológica George Dahl.

Carbones del Cerrejón Limited. (2010). Programa de conservación del Kayuüshi (Crocodylus acutus) y tortugas marinas en la Alta Guajira - Colombia
(Informe). Barranquilla: Fundación Hidrobiológica George Dahl.

Carbones del Cerrejón Limited. (2011). Programa de conservación de especies amenazadas en la Alta Guajira - Colombia (Informe). Barranquilla: Fundación Hidrobiológica George Dahl.

Casas-Andreu, G. (2003). Ecología de la anidación de Crocodylus acutus (Reptilia: Crocodylidae) en la desembocadura del río Cuitzmala, Jalisco, México. Acta Zoológica Mexicana, 89, 111-128.

Charruau, P., Thorbjarnarson, J., \& Hénaut, Y. (2010). Tropical cyclones and reproductive ecology of Crocodylus acutus Cuvier, 1807 (Reptilia: Crocodilia: Crocodylidae) on a Caribbean atoll in México. Journal of Natural History, 44, 741-761.

Charruau, P. (2012). Microclimate of American crocodile nests in Banco Chinchorro biosphere reserve, México: Effect on incubation length, embryos survival and hatchlings sex. Journal of Thermal Biology, 37, 6-14.

CORPOGUAJIRA. (2006). Programa de conservación del Crocodylus acutus (Caimán aguja) en Bahía Portete, Departamento de la Guajira (Informe). Convenio 0249. De 2005, Asociación Desarrollo Guajiro, Riohacha.

Cupul-Magaña, F. G., De Niz-Villaseñor, A., Reyes-Juárez, A., \& Rubio-Delgado, A. (2004). Historia natural del Cocodrilo Americano (Crocodylus acutus) en el estero Boca Negra, Jalisco, México: anidación y crecimiento de neonatos. Ciencia y Mar, 23, 31-42.

CVS - Corporación Autónoma Regional de los Valles del Sinú y del San Jorge. (2012). Proyecto de conservación del Crocodylus acutus de la bahía de Cispatá con la participación de las comunidades locales municipio de San Antero -Departamento de Córdoba caribe de Colombia. Examen de las propuestas de enmienda a los apéndices i y ii. Decimo-sexta reunión de la Conferencia de las Partes Bangkok (Tailandia) 3 al 14 de marzo 2012. Cop16 Prop.

De la Hoz, D., Patiño, E., Gómez, J., Mejía, F., \& Báez, L. (2007). Population diagnosis and some reproductive aspect of the American crocodiles (Crocodylus acutus) in Portete Bay-Guajira peninsular-Colombia. In IUCN-CSG Crocodiles (Eds.), Proceeding of the 19th Working Meeting of Crocodiles Specialist Group (pp. 450-466). Gland, Switzerland: IUCN.

De La Ossa-Lacayo, A., De La Ossa, V. J., Fajardo Patiño, A., \& Morales-Betancourt, M. A. (2013). Crocodylus acutus. En M. A. Morales-Betancourt, C. A. Lasso, J. De La Ossa, \& A. Fajardo-Patiño (Eds.), VIII. Biología y conservación de los Crocodylia de Colombia (pp. 93-108). Serie Editorial Recursos Hidrobiológicos y Pesqueros Continentales de Colombia. Instituto de Investigación de Recursos Biológicos Alexander von Humboldt (IAvH). Bogotá, D.C., 
Colombia: JAVEGARAF-Fundación Cultural Javeriana de Artes Gráficas.

Dunson, W. A. (1982). Salinity relations of crocodiles in Florida Bay. Copeia, 2, 374-385.

Dunson, W. A., \& Mazzotti, F. J. (1989). Salinity as a limiting factor in the distribution of reptiles in Florida Bay: a theory for the estuarine origin of marine snakes and turtles. Bulletin of Marine Science, 44, 229-244.

Espinosa-Blanco, A., Seijas, A. E., \& Hernández, O. (2010). Reproducción y colecta de huevos del caimán del Orinoco (Crocodylus intermedius) en el sistema del río Cojedes, Venezuela. En IUCN-CSG Crocodiles (Eds.), Crocodiles. Proceedings of the 20th Working Meeting of the Crocodile Specialist Group (pp. 32-40). Gland, Switzerland and Cambridge UK: IUCN.

Espinosa-Blanco, A., Seijas, A. E., \& Hernández, O. (2013). Egg collection as a conservation tool of Orinoco Crocodile (Crocodylus intermedius) in the Cojedes River System, Venezuela. Interciencia, 38, 358-363.

Ferguson, M. (1985). Reproductive Biology and Embryology of the Crocodilians. In C. Gans, F. S. Billett, \& P. F. A. Maderson. Biology of the Reptilia (vol. 14, pp. 329-491). New York, USA: Wiley and Sons.

Fox, J. (2005). The R Commander: A Basic Statistics Graphical User Interface to R. Journal of Statistical Software, 14, 1-42.

Galindo, G., Marcelo, D., Bernal, N. R., Vergara, L. K., \& Betancourth, J. C. (2009). Planificación ecorregional para la conservación de la biodiversidad en el Caribe continental colombiano. Serie Planificación Ecoregional para la Conservación de la Biodiversidad, No. 1. Bogotá D.C., Colombia: Instituto de Investigación de Recursos Biológicos Alexander Von Humboldt, Agencia Nacional de Hidrocarburos, The Nature Conservancy e Instituto de Hidrología, Meteorología y Estudios Ambientales.

Gil-Torres, W., Fonseca, G. J., Restrepo, P., Figueroa, L., Gutiérrez, G., Gómez, M., Sierra-Correa, P. C., Hernández-Ortiz, M., López, A., \& Segura-Quintero, C. (2009). Ordenamiento ambiental de los manglares de la Alta, Media y Baja Guajira. La Guajira, Colombia: Instituto de Investigaciones Marinas y Costeras José Benito Vives De Andréis, Corporación Autónoma Regional de la Guajira, Ministerio de Ambiente vivienda y Desarrollo Territorial.

Gómez, J. \& Patiño, E. (2010). Programa de conservación de caimanes y babillas con la participación y concertación comunitaria, en el sector Bahía Hondita y en los humedales costeros existentes, entre los ríos Ranchería y Palomino, departamento de La Guajira, Colombia (Informe). Riohacha: CORPOGUAJIRA.
Greer, A. E. (1975). Clutch Size in Crocodilians. Journal of Herpetology, 9, 319-322.

Grigg, G. (1987). Water relations of crocodilian eggs: Management considerations. In J. W. Grahamel, S. Webb, C. Manolis, \& J. Whitehead (Eds.), Wildlife management crocodiles and alligators ( $\mathrm{pp} 499$ 502). Sydney, Australia: Surrey Beatty and Sons Pty Limited.

Gutiérrez-Moreno, C., Alonso, D., \& Segura-Quintero, C. (2008). Diseño de un área marina protegida para Bahía Portete-la Guajira, Caribe colombiano. Boletín Investigaciones Marinas y Costeras, 37, 189-212.

Hernández-Camacho, J., Hurtado-Guerra, A., Ortiz-Quijano, R., \& Walschburger, T. (1992). Unidades biogeográficas de Colombia. En G. Halffter (Ed.), La diversidad biológica de Iberoamérica (pp. 105151). México D.F., México: I. CYTED-B, Programa Iberoamericano de Ciencia y Tecnología para el Desarrollo, Instituto de Ecología, A.C. Secretaría de Desarrollo Social.

Hernández-Hurtado, H., Romero-Villaruel, J. J., \& Hernández-Hurtado, P. S. (2011). Ecología poblacional de Crocodylus acutus en los sistemas estuarinos de San Blas, Nayarit, México. Revista Mexicana de Biodiversidad, 82, 887-895.

Hernández-Hurtado, P., Vega-Villasante, F., HernándezHurtado, H., Cupul-Magaña, F. G., \& García de Quevedo-Machain, R. (2013). Éxito de eclosión por incubación artificial en nidos de Crocodylus acutus (Reptilia: Crocodylia) en cautiverio. Cuadernos de Herpetología, 27, 71-75.

Huchzermeyer, F. W. (2003). Crocodiles: Biology, Husbandry and Diseases. Cambridge, USA: CABI Publishing of CAB International.

Hutton, J. M., \& Webb, G. J. W. (1992). An introduction to the farming of crocodilians. En R. A. Luxmoore (Ed.), Directory of crocodilian farming operations (pp. 1-39). Gland \& Cambridge, United Kingdom: IUCN.

Instituto Geográfico Agustín Codazzi. (2014). Diccionario geográfico de Colombia en la web. Recuperado de http://www.igac.gov.co/digeo/app/index.html

Kushlan, J., \& Mazzotti, F. J. (1989). Population Biology of the American Crocodile. Journal of Herpetology, $23,7-21$.

Larriera, A., \& Imhof, A. (2004). Caiman yacare and Caiman latirostris Ranching Programs in Argentina. En IUCN-CSG Crocodiles (Eds.), Crocodiles. Proceedings of the 17th Working Meeting of the Crocodile Specialist Group (pp. 216-219). Gland, Switzerland: UICN - The World Conservation Union.

Lutz, P. L., \& Dunbar-Cooper, A. (1984). The Nest Environment of the American Crocodile (Crocodylus acutus). Copeia, 1, 153-161. 
Mazzotti, F. (1983). The ecology the Crocodylus acutus in Florida (Tesis de doctorado). The Pennsylvania State University, State College.

Mazzotti, F., Bohnsack, B., McMahon, M., \& Roos, J. (1986). Field and laboratory observations on the effects of high temperature and salinity on hatchling Crocodylus acutus. Herpetologica, 42, 191-196.

Mazzotti, F. (1989). Factor affecting the nesting success of the American Crocodiles, Crocodylus acutus, in Florida Bay. Bulletin of Marine Science, 44, 220-228.

Mazzotti, F. (1999). The American crocodile in Florida Bay. Estuaries, 22, 552-561.

Medem, F. (1981). Los Crocodylia de Sur América. Los Crocodylia de Colombia. Bogotá, Colombia: Editorial Carrera $7 \mathrm{a}$ Ltd.

Medem, F. (1983). Los Crocodylia de Sur América. Venezuela, Trinidad, Tobago, Guyana, Surinam, Guyana Francesa, Ecuador, Perú, Bolivia, Brasil, Paraguay, Argentina y Uruguay. Bogotá, Colombia: Editorial Carrera 7a Ltd.

Medina, H., García, J., \& Núñez, D. (2007). El método del hidrómetro: base teórica para su empleo en la determinación de la distribución del tamaño de partículas de suelo. Revista Ciencias Técnicas Agropecuarias, $16,19-24$.

Ministerio de Ambiente y Desarrollo Sostenible. (2014). Por medio del cual se declara, reserva, delimita y alindera el Parque Nacional Natural Bahía Portete - Kaurrele. Resolución. 2096 de 19 de diciembre de 2014.

Pérez, O. \& Escobedo-Galván, A. H. (2005a). Observaciones biométricas de Crocodylus acutus (Cuvier, 1807) recién nacidos en cautiverio, Tumbes, Perú. Revista Peruana de Biología, 12, 171-172.

Pérez, O. \& Escobedo-Galván, A. H. (2005b). Notas sobre la reproducción en cautiverio de Crocodylus acutus (Cuvier, 1807) en el Perú. Revista Peruana de Biología, 12, 479-481.

Platt, S. G., \& Thorbjarnarson, J. B. (2000). Nesting ecology of the American crocodile in the coastal zone of Belize. Copeia, 3, 868-873.

Ponce-Campos, P., Thorbjarnarson, J., \& Velasco, A. (2012). Crocodylus acutus. The IUCN Red List of Threatened Species. Version 2014.3. <www.iucnredlist.org>. Downloaded on 25 March 2015.

R Development Core Team. (2011). R: A language and environment for statistical computing. R Foundation for Statistical Computing, Viena. http://www.R-project.org/. 13/04/2011

Richards, P. M., Mooij, W. M., \& De Angelis, D. L. (2004). Evaluating the effect of salinity on a simulated American crocodile (Crocodylus acutus) population with applications to conservation and Everglades restoration. Ecological Modelling, 180, 371-394.

Rodríguez-Melo, M. (2000a). Cocodrilos (Archosauria: Crocodylia) de la región Neotropical. Biota Colombiana, 1, 135-140.

Rodríguez-Melo, M. (2000b). Estado y distribución de los Crocodylia en Colombia. Compilación de resultados del Censo Nacional, 1994 a 1997. Bogotá, Colombia: Ministerio del Medio Ambiente, Instituto de Investigaciones Alexander Von Humboldt.

Rodríguez-Melo, M. (2002). Crocodylus acutus. En O. V. Mora-Castaño (Ed.), Libro rojo de reptiles de Colombia. Libros rojos de especies amenazadas de Colombia (pp. 41-48). Bogotá, Colombia: Instituto de Ciencias Naturales, Universidad Nacional de Colombia, Ministerio del Medio Ambiente, Conservación Internacional.

Rodríguez-Soberon, R. (2000). Situación actual del Crocodylus acutus en Cuba. En IUCN-CSG Crocodiles (Eds.), Proceedings of the 15th Working Meeting of the Crocodile Specialist Group (pp. 17-41). Gland, Switzerland and Cambrige UK: IUCN - The World Conservation Union.

Rodríguez-Soberon, R., Tabet, M., \& Berovides, V. (2002). Nidificación del Cocodrilo Americano (Crocodylus acutus Cuvier) en el Refugio de fauna "Monte Montecabanigua", Cuba. En M. L. Verada \& L. Larriera (Eds.), La conservación y manejo de Caimanes y Cocodrilos de América Latina (Vol 2, pp. 135-156.). Sao Paulo, Brasil: CN Editorial.

Ross, J. P. 1998. Crocodiles. Status Survey and Conservation Action Plan (2nd Edition). Switzerland and Cambridge, UK: IUCN/SSC Crocodile Specialist Group, IUCN.

Rueda-Almonacid, J. V., Carr, J. L., Mittermeir, R. A., Rodríguez-Mahecha, J. V., Mast, R. B., Vogt, R. C., Rhodin, A. G., Ossa-Velásquez, J., Rueda, J. N., \& Mittermeier, C. G. (2007). Las tortugas y cocodrilianos de los países andinos del trópico. Serie de guías tropicales de campo No 6. Conservación Internacional. Bogotá, Colombia: Editorial Panamericana, Formas e Impresos.

Sánchez-Páez, H., Ulloa-Delgado, G., Álvarez-León, R. Gil-Torres, O., Sánchez-Alférez, A., Guevara-Mancera, O., Patiño-Callejas, L., \& Páez-Parra, F. (2000). Hacia la recuperación de los manglares del Caribe colombiano. Bogotá, Colombia: Ministerio del Medio Ambiente. Asociación Colombiana de Reforestadores (ACOFORE). Organización Internacional de Maderas Tropicales (OIMT).

Sánchez-Páez, H., Ulloa-Delgado, G., \& Arsenio-Tavera, H. A. (2004). Manejo integral de manglares por comunidades locales - Caribe de Colombia. Bogotá, Colombia: Ministerio de Medio Ambiente Vivienda y Desarrollo Territorial, Corporación Nacional 
de Investigación Fomento Forestal, Organización Internacional de Maderas Tropicales.

Seijas, A. E., Urdaneta, A., \& Barros, T. (2008). Tasas de crecimiento durante primer año de vida y la temporada de eclosión de caimán de la costa (Crocodylus acutus) en Venezuela. Boletín del Centro de Investigaciones Biológicas, 42, 445-459.

Seijas, A. E. (2011). Los Crocodylia de Venezuela. Academia de Ciencias Físicas, Matemáticas y Naturales. Ecología y Conservación. Colección Estudios y Divulgación Científica y Tecnológica. Venezuela: Brima Color.

Sigler, L. (2010). La historia natural del Cocodrilo Americano Crocodylus acutus en el Parque Nacional Cañón del Sumidero, Chiapas, México. Revista Latinoamericana de Conservación, 1, 73-82.

Solano, O. (1994). Corales, formaciones arrecifales y blanqueamiento de 1987 en Bahía Portete (Guajira, Colombia). Anales Instituto de Investigaciones Marinas Punta Betín, 23, 149-163.

Schurbert, A. (2002). Reproducción del Cocodrilo Americano (Crocodylus acutus) en el lago Enriquillo, Republica Dominicana. In IUCN-CSG Crocodiles (Eds.), Crocodiles. Proceeding of the $16^{\text {th }}$ working meeting of the Crocodile Specialist Group (pp. 244-252.) Gland, Switzerland and Cambridge UK: IUCN - The World Conservation Union.

Thorbjarnarson, J. (1988). The status and ecology of the American crocodile in Haiti. Bull. Florida State Bulletin Florida State Museum. Biological Sciences, $33,1-86$

Thorbjarnarson, J. (1989). Ecology of the American Crocodile (Crocodylus acutus). In IUCN-CSG Crocodiles (Eds.), Crocodiles: their Ecology, Management and Conservation (pp. 228-259). Switzerland: Special Pub. Croc. Spec. Group. IUCN- The World Conservation Union Publ. N. S.

Thorbjarnarson, J. (1996). Reproductive characteristics of the order crocodylia. Herpetology, 52, 8-24.

Thorbjarnarson, J. (2001). Manejo sustentable de caimanes y cocodrilos. En R. Primack, R. Roíz, P. Feinsinger, R. Dirzo, \& F. Massarado (Eds.), Fundamentos de conservación biológica perspectivas latinoamericanas (pp. 541-543). México D.F., México: Fondo de Cultura Económica.

Thorbjarnarson, J., Mazzotti, F., Sanderson, F., Buitrago, F., Lazcano, M., Minkowski, K., Muñiz, M., Ponce, P., Sigler, L., Soberon, R., Trelancia, A. M., \& Velasco, A. (2006). Regional habitat conservation priorities for the American crocodile. Biological Conservation, $12,25-36$.
Thorbjarnarson, J. (2010). American crocodile (Crocodylus acutus). En S. C. Manolis, \& C. Stevenson (Eds.), Crocodiles. Status survey and conservation action plan (Tercera edición, pp. 46-53). Darwin: Crocodile Specialist Group. http://www.iucncsg.org/pages/ Publications.html. 06/02/2011

Towsend, W. (2003). La investigación participativa y su utilidad para el manejo de la fauna silvestre en Bolivia. En C. Campos-Roso \& A. Ulloa (Eds.), Fauna socializada "Tendencias en el manejo participativo de la fauna en América Latina” (pp. 117-129). Bogotá, Colombia: Editorial Fundación Natura, Mac Arthur Foundation, Instituto Colombiano de Antropología.

Ulloa-Delgado, G. A., Sierra-Díaz, C. L., \& Cavanzo, D. (2005). Proyecto de conservación del Crocodylus acutus (Cuvier, 1807) y su hábitat natural - bahía de Cispatá departamento de Córdoba-Colombia. En IUCN-CSG Crocodiles (Eds.), Proceedings de la Reunión Regional de América Latina y el Caribe del Grupo de Especialistas en Cocodrilos. Santa Fe, Argentina: Crocodile Specialist Group, Species Survival Commission, International Union for Conservation of Nature.

Ulloa-Delgado, G. A., \& Sierra-Díaz, C. (2006). Experimental pilot project for the conservation of Crocodylus acutus by local communities in the mangrove of Cispatá Bay, Cordoba, Colombia, South America. En IUCN-CSG Crocodiles (Eds.), Crocodiles. Proceedings of the 18th Working Meeting of the Crocodile Specialist Group (pp. 156-164). Gland, Switzerland and Cambridge UK: IUCN - The World Conservation Union.

Ulloa-Delgado, G. A. (2011). Natural populations of Crocodiles discovered in the Catatumbo region. CSG Newsletter, 30, 6 .

Ulloa-Delgado, G. A., \& Sierra-Díaz, C. L. (2012). Proyecto de conservación del Crocodylus acutus de la Bahía de Cispatá con la participación de las comunidades locales. Documento de apoyo técnico para la solitud de la Enmienda del Apéndice 1 al 2 de la CITES. Municipio de San Antero- Departamento de Córdoba. Caribe de Colombia: Corporación Autónoma Regional de los valles del Sinú y del San Jorge CVS- Ministerio de Ambiente y Desarrollo Sostenible MADS- Instituto de investigaciones Biológicas Alexander Von Humboldt IAVH y Asociación comunitaria para la conservación del caimán de la Bahía de Cispatá- ASOCAIMAN.

Webb, G., Manolis, C., \& Whitehead, J. (1987). Wildlife management: Crocodiles and alligators. Surrey Sydney, Australia: Surrey Beatty and Sons Pty Limited.

Wickham, H. (2009). ggplot2: elegant graphics for data analysis. New York, USA: Springer. 